\title{
$A$-statistical convergence of Mittag-Leffler operators
}

\author{
Mehmet Ali Özarslan
}




\title{
$A$-STATISTICAL CONVERGENCE OF MITTAG-LEFFLER OPERATORS
}

\author{
MEHMET ALI ÖZARSLAN
}

Received February 21, 2012

\begin{abstract}
In this paper we introduce the Mittag-Leffler operators, which includes the modified Szász-Mirakjan operators. We obtain the transformation properties and compute the rate of convergence by using modulus of continuity. Furthermore we give the $A$-statistical approximation theorem for these operators.
\end{abstract}

2000 Mathematics Subject Classification: 41A25; 41A36

Keywords: Mittag-Leffler operators, Szász-Mirakjan operators, A-statistical convergence and statistical convergence, modulus of continuity, Bernoulli numbers

\section{INTRODUCTION}

The function defined by [11]

$$
E_{\alpha}(z)=\sum_{k=0}^{\infty} \frac{z^{k}}{\Gamma(\alpha k+1)} \quad(z \in \mathbb{C} ; \mathcal{R}(\alpha)>0)
$$

is known as the Mittag-Leffler function. The two-index Mittag-Leffler function is defined by [14]

$$
E_{\alpha, \beta}(z)=\sum_{k=0}^{\infty} \frac{z^{k}}{\Gamma(\alpha k+\beta)} \quad(z, \beta \in \mathbb{C} ; \mathcal{R}(\alpha)>0) .
$$

Note that $E_{\alpha, 1}(z)=E_{\alpha}(z)$ and

$$
E_{1,1}(z)=e^{z}, E_{1,2}(z)=\frac{e^{z}-1}{z}, E_{1, m+1}(z)=\frac{e^{z}-\sum_{k=0}^{m-1} \frac{x^{k}}{k !}}{z^{m}} .
$$

Moreover, for $|z|<2 \pi$, we have

$$
\frac{1}{E_{1,2}(z)}=\sum_{n=0}^{\infty} B_{n} \frac{z^{n}}{n !}, \quad \frac{1}{E_{1, m+1}(z)}=\sum_{n=0}^{\infty} B_{n}^{(m)} \frac{z^{n}}{n !}
$$


where the coefficients $\left(B_{n}\right)$ are the familiar Bernoulli numbers and $\left(B_{n}^{(m)}\right)$ are the generalized Bernoulli numbers (see [2]).

Let $\left(b_{n}\right)$ be a sequence of positive real numbers and let $\beta>0$ be fixed. For all $n \in \mathbb{N}$, we introduce the Mittag-Leffler operators by

$$
L_{n}^{(\beta)}(f ; x)=\frac{1}{E_{1, \beta}\left(\frac{n x}{b_{n}}\right)} \sum_{k=0}^{\infty} f\left(\frac{k}{n} b_{n}\right) \frac{(n x)^{k}}{b_{n}^{k} \Gamma(k+\beta)},
$$

where $f \in E:=\left\{f \in C[0,+\infty): \lim _{x \rightarrow+\infty} \frac{f(x)}{1+x^{2}}\right.$ is finite $\}$ and $C[0,+\infty)$ denotes the space of continuous functions defined on $[0,+\infty)$. Recall that the Banach lattice $E$ is endowed with the norm

$$
\|f\|_{*}:=\sup _{x \in[0,+\infty)} \frac{|f(x)|}{1+x^{2}} .
$$

It is obvious that the operators $L_{n}^{(\beta)}(f ; x)$ defined in (1.1) are linear and positive.

Note that for $\beta=1$, we have

$$
L_{n}^{(1)}(f ; x)=e^{-n x / b_{n}} \sum_{k=0}^{\infty} f\left(\frac{k}{n} b_{n}\right) \frac{(n x)^{k}}{b_{n}^{k} k !}=S_{n}(f ; x)
$$

where the operators $S_{n}$ are the modified Szász-Mirakjan operators considered in [1].

By direct computations one can state the following lemma;

Lemma 1. Let $\psi_{x}^{2}(t)=(t-x)^{2}$. Then, for each $x \geq 0$ and $n \in \mathbb{N}$, we have

(a) $L_{n}^{(\beta)}(1 ; x)=1$,

(b) $\left|L_{n}^{(\beta)}(t ; x)-x\right| \leq \frac{|1-\beta| b_{n}}{n}$,

(c)

$$
\begin{aligned}
\left|L_{n}^{(\beta)}\left(t^{2} ; x\right)-x^{2}\right| \leq & \frac{(2|1-\beta|+1) b_{n}}{n} x \\
& +\frac{\left(2(1-\beta)^{2}+|1-\beta|+|1-\beta||\beta-2|\right) b_{n}^{2}}{n^{2}}
\end{aligned}
$$

(d)

$$
\begin{aligned}
L_{n}^{(\beta)}\left(\psi_{x}^{2} ; x\right) \leq & \frac{(4|1-\beta|+1) b_{n}}{n} x \\
& +\frac{\left(2(1-\beta)^{2}+|1-\beta|+|1-\beta||\beta-2|\right) b_{n}^{2}}{n^{2}} .
\end{aligned}
$$


Proof. Since

$$
\sum_{k=0}^{\infty} \frac{(n x)^{k}}{b_{n}^{k} \Gamma(k+\beta)}=E_{1, \beta}\left(\frac{n x}{b_{n}}\right),
$$

then $L_{n}^{(\beta)}(1 ; x)=1$. Using the fact that $\Gamma(k+\beta)=(k+\beta-1) \Gamma(k+\beta-1)$, we get

$$
\begin{aligned}
L_{n}^{(\beta)}(t ; x) & =\frac{1}{E_{1, \beta}\left(\frac{n x}{b_{n}}\right)} \sum_{k=1}^{\infty} \frac{k b_{n}}{n} \frac{(n x)^{k}}{b_{n}^{k} \Gamma(k+\beta)} \\
& =\frac{1}{E_{1, \beta}\left(\frac{n x}{b_{n}}\right)} \sum_{k=1}^{\infty} \frac{[(k+\beta-1)+1-\beta] b_{n}}{n} \frac{(n x)^{k}}{b_{n}^{k}(k+\beta-1) \Gamma(k+\beta-1)} \\
& =x+\frac{1}{E_{1, \beta}\left(\frac{n x}{b_{n}}\right)} \sum_{k=1}^{\infty} \frac{1-\beta}{n} \frac{b_{n}(n x)^{k}}{b_{n}^{k} \Gamma(k+\beta)} .
\end{aligned}
$$

Hence

$$
\left|L_{n}^{(\beta)}(t ; x)-x\right|=\frac{|1-\beta| b_{n}}{n} \frac{1}{E_{1, \beta}\left(\frac{n x}{b_{n}}\right)} \sum_{k=1}^{\infty} \frac{(n x)^{k}}{b_{n}^{k} \Gamma(k+\beta)} \leq \frac{|1-\beta| b_{n}}{n} .
$$

Similarly, by $k(k-1)=(k+\beta-1)(k+\beta-2)+2(1-\beta) k+(1-\beta)(\beta-2)$ and $\Gamma(k+\beta)=(k+\beta-1)(k+\beta-2) \Gamma(k+\beta-2)$, we get

$$
\begin{aligned}
L_{n}^{(\beta)}\left(t^{2} ; x\right) & =\frac{1}{E_{1, \beta}\left(\frac{n x}{b_{n}}\right)} \sum_{k=1}^{\infty}\left(\frac{k}{n} b_{n}\right)^{2} \frac{(n x)^{k}}{b_{n}^{k} \Gamma(k+\beta)} \\
& =\frac{1}{E_{1, \beta}\left(\frac{n x}{b_{n}}\right)} \sum_{k=1}^{\infty} \frac{(k(k-1)+k) b_{n}^{2}}{n^{2}} \frac{(n x)^{k}}{b_{n}^{k} \Gamma(k+\beta)} \\
& =\frac{1}{E_{1, \beta}\left(\frac{n x}{b_{n}}\right)} \sum_{k=2}^{\infty} \frac{k(k-1) b_{n}^{2}}{n^{2}} \frac{(n x)^{k}}{b_{n}^{k} \Gamma(k+\beta)}+\frac{b_{n} L_{n}^{(\beta)}(t ; x)}{n} \\
= & \frac{1}{E_{1, \beta}\left(\frac{n x}{b_{n}}\right)} \sum_{k=2}^{\infty} \frac{(k+\beta-1)(k+\beta-2) b_{n}^{2}}{n^{2}} \\
& \times \frac{(n x)^{k}}{b_{n}^{k}(k+\beta-1)(k+\beta-2) \Gamma(k+\beta-2)}
\end{aligned}
$$




$$
\begin{aligned}
& +\frac{1}{E_{1, \beta}\left(\frac{n x}{b_{n}}\right)} \sum_{k=2}^{\infty} \frac{2(1-\beta) k b_{n}^{2}}{n^{2}} \frac{(n x)^{k}}{b_{n}^{k} \Gamma(k+\beta)} \\
& +\frac{(1-\beta)(\beta-2) b_{n}^{2}}{n^{2} E_{1, \beta}\left(\frac{n x}{b_{n}}\right)} \sum_{k=2}^{\infty} \frac{(n x)^{k}}{b_{n}^{k} \Gamma(k+\beta)}+\frac{b_{n} L_{n}^{(\beta)}(t ; x)}{n} .
\end{aligned}
$$

Therefore

$$
\begin{aligned}
\left|L_{n}^{(\beta)}\left(t^{2} ; x\right)-x^{2}\right| \leq & \frac{2|1-\beta| b_{n}}{n E_{1, \beta}\left(\frac{n x}{b_{n}}\right)} \sum_{k=2}^{\infty} \frac{k b_{n}}{n} \frac{(n x)^{k}}{b_{n}^{k} \Gamma(k+\beta)} \\
& +\frac{|1-\beta||\beta-2| b_{n}^{2}}{n^{2} E_{1, \beta}\left(\frac{n x}{b_{n}}\right)} \sum_{k=2}^{\infty} \frac{(n x)^{k}}{b_{n}^{k} \Gamma(k+\beta)}+\frac{b_{n}\left|L_{n}^{(\beta)}(t ; x)\right|}{n} \\
\leq & \frac{(2|1-\beta|+1) b_{n}}{n}\left|L_{n}^{(\beta)}(t ; x)\right|+\frac{|1-\beta||\beta-2| b_{n}^{2}}{n^{2}} .
\end{aligned}
$$

Using (1.2), we obtain

$$
\begin{aligned}
\left|L_{n}^{(\beta)}\left(t^{2} ; x\right)-x^{2}\right| \leq & \frac{(2|1-\beta|+1) b_{n}}{n} x \\
& +\frac{\left(2(1-\beta)^{2}+|1-\beta|+|1-\beta||\beta-2|\right) b_{n}^{2}}{n^{2}}
\end{aligned}
$$

Finally,

$$
\begin{aligned}
& L_{n}^{(\beta)}\left(\psi_{x}^{2} ; x\right) \\
& \leq\left|L_{n}^{(\beta)}\left(t^{2} ; x\right)-x^{2}\right|+2 x\left|L_{n}^{(\beta)}(t ; x)-x\right|+x^{2}\left|L_{n}^{(\beta)}(1 ; x)-1\right| \\
& \leq \frac{(4|1-\beta|+1) b_{n}}{n} x+\frac{\left(2(1-\beta)^{2}+|1-\beta|+|1-\beta||\beta-2|\right) b_{n}^{2}}{n^{2}}
\end{aligned}
$$

which completes the proof.

We organize the paper as follows: In Section 2, we give the transformation properties of the operators $L_{n}^{(\beta)}$ and compute the rate of convergence by using the modulus of continuity. In Section 3, we prove an $A$-statistical Korovkin type approximation theorem.

\section{TRAnSFormation PROPERTIES AND RATE of CONVERgEnCE}

We start with the following lemma, which proves that $L_{n}^{(\beta)}$ maps $E$ into itself. 
Lemma 2. Let $\left(\frac{b_{n}}{n}\right)$ be a bounded sequence of positive numbers and $\beta>0$ be fixed. Then there exists a constant $M(\beta)$ such that, for $w(x)=\left(1+x^{2}\right)^{-1}$, we have

$$
w(x) L_{n}^{(\beta)}\left(\frac{1}{w} ; x\right) \leq M(\beta)
$$

holds for all $x \in[0, \infty)$ and $n \in \mathbb{N}$. Furthermore, for all $f \in E$, we have

$$
\left\|L_{n}^{(\beta)}(f)\right\|_{*} \leq M(\beta)\|f\|_{*} .
$$

Proof. Using Lemma 1, we can write that

$$
\begin{aligned}
w(x) L_{n}^{(\beta)}\left(\frac{1}{w} ; x\right)= & \frac{1}{1+x^{2}}\left[L_{n}^{(\beta)}(1 ; x)+L_{n}^{(\beta)}\left(t^{2} ; x\right)\right] \\
\leq & \frac{1}{1+x^{2}}\left[1+x^{2}+\frac{(2|1-\beta|+1) b_{n}}{n} x\right. \\
& \left.+\frac{\left(2(1-\beta)^{2}+|1-\beta|+|1-\beta||\beta-2|\right) b_{n}^{2}}{n^{2}}\right] \\
\leq & M(\beta) .
\end{aligned}
$$

On the other hand

$w(x)\left|L_{n}^{(\beta)}(f ; x)\right|=w(x)\left|L_{n}^{(\beta)}\left(w \frac{f}{w} ; x\right)\right| \leq\|f\|_{*} w(x) L_{n}^{(\beta)}\left(\frac{1}{w} ; x\right) \leq M(\beta)\|f\|_{*}$.

Taking supremum over $x \in[0, \infty)$ in the above inequality, gives the result.

Now, recall that the usual modulus of continuity of $f$ on the closed interval $[0, B]$ is defined by

$$
\omega_{B}(f, \delta)=\sup _{\substack{|t-x| \leq \delta \\ x, t \in[0, B]}}|f(t)-f(x)| .
$$

It is well known that, for a function $f \in E$, we have $\lim _{\delta \rightarrow \infty} \omega_{B}(f, \delta)=0$.

The next theorem gives the rate of convergence of the operators $L_{n}^{(\beta)}(f ; x)$ to $f(x)$, for all $f \in E$.

Theorem 1. Let $\beta>0$ be fixed, $\left(\frac{b_{n}}{n}\right)$ be a bounded sequence of positive numbers, $f \in E$ and $\omega_{B+1}(f, \delta)(B>0)$ be its modulus of continuity on the finite interval $[0, B+1] \subset[0, \infty)$. Then

$$
\left\|L_{n}^{(\beta)}(f ; x)-f(x)\right\|_{C[0, B]} \leq M_{f}(\beta, B) \delta_{n}(\beta, B)+2 \omega_{B+1}\left(f, \delta_{n}^{1 / 2}(\beta, B)\right)
$$


where $\delta_{n}(\beta, B)=N_{f}(\beta, B) \frac{b_{n}}{n}\left[1+\frac{b_{n}}{n}\right]$,

$$
N_{f}(\beta, B)=\max \left\{(4|1-\beta|+1) B,\left(2(1-\beta)^{2}+|1-\beta|+|1-\beta||\beta-2|\right)\right\}
$$

and $M_{f}(\beta, B)$ is an absolute constant depending on $f, \beta$ and $B$.

Proof. Let $\beta>0$ be fixed. For $x \in[0, B]$ and $t \leq B+1$, we have the inequality

$$
|f(t)-f(x)| \leq \omega_{B+1}(f,|t-x|) \leq\left(1+\frac{|t-x|}{\delta}\right) \omega_{B+1}(f, \delta)
$$

where $\delta>0$. On the other hand, for $x \in[0, B]$ and $t>B+1$, using the fact that $t-x>1$, we have

$$
|f(t)-f(x)| \leq A_{f}\left(1+x^{2}+t^{2}\right) \leq A_{f}\left(2+3 x^{2}+2(t-x)^{2}\right) \leq 6 A_{f}\left(1+B^{2}\right)(t-x)^{2}
$$

By (2.1) and (2.2), we get for all $x \in[0, B]$ and $t \geq 0$ that

$$
|f(t)-f(x)| \leq 6 A_{f}\left(1+B^{2}\right)(t-x)^{2}+\left(1+\frac{|t-x|}{\delta}\right) \omega_{B+1}(f, \delta) .
$$

Therefore

$$
\begin{gathered}
\left|L_{n}^{(\beta)}(f ; x)-f(x)\right| \\
\leq 6 A_{f}\left(1+B^{2}\right) L_{n}^{(\beta)}\left((t-x)^{2} ; x\right)+\left(1+\frac{L_{n}^{(\beta)}(|t-x| ; x)}{\delta}\right) \omega_{B+1}(f, \delta) .
\end{gathered}
$$

Applying Cauchy-Schwarz inequality and Lemma 1, we get

$$
\left.\begin{array}{c}
\quad\left|L_{n}^{(\beta)}(f ; x)-f(x)\right| \\
\leq 6 A_{f}\left(1+B^{2}\right) L_{n}^{(\beta)}\left(\psi_{x}^{2} ; x\right)+\left(1+\frac{\left[L_{n}^{(\beta)}\left(\psi_{x}^{2} ; x\right)\right]^{1 / 2}}{\delta}\right) \omega_{B+1}(f, \delta) \\
\leq 6 A_{f}\left(1+B^{2}\right) \\
+\left(\begin{array}{c}
\times\left[(4|1-\beta|+1) B \frac{b_{n}}{n}+\left(2(1-\beta)^{2}+|1-\beta|+|1-\beta||\beta-2|\right) \frac{b_{n}^{2}}{n^{2}}\right] \\
1+
\end{array}\right. \\
\left.\quad \frac{\left[(4|1-\beta|+1) B \frac{b_{n}}{n}+\left(2(1-\beta)^{2}+|1-\beta|+|1-\beta||\beta-2|\right) \frac{b_{n}^{2}}{n^{2}}\right]^{1 / 2}}{\delta}\right) \\
\times \omega_{B+1}(f, \delta) \leq M_{f}(\beta, B) \delta_{n}(\beta, B)+2 \omega_{B+1}\left(f,\left(\delta_{n}(\beta, B)\right)^{1 / 2}\right),
\end{array}\right)
$$


where

$$
N_{f}(\beta, B)=\max \left\{(4|1-\beta|+1) B,\left(2(1-\beta)^{2}+|1-\beta|+|1-\beta||\beta-2|\right)\right\},
$$

$M_{f}(\beta, B)=6 A_{f}\left(1+B^{2}\right)$ and $\delta_{n}(\beta, B)=N_{f}(\beta, B) \frac{b_{n}}{n}\left[1+\frac{b_{n}}{n}\right]$. Whence the result follows.

\section{3. $A$-STATistical CONVERGENCE}

Recently, $A$-statistical convergence of linear positive operators have been an active research area (see [3-5,12]). We start to this section by recalling concepts of $A$ statistical convergence.

Let $A=\left(a_{j k}\right)$ be a non-negative regular summability matrix.

Definition 1. The $A$-density of a subset $K$ of $\mathbb{N}$ is given by

$$
\delta_{A}(K)=\lim _{j} \sum_{k \in K} a_{j, k},
$$

provided that limit exists (see [7]).

Definition 2. A sequence $x=\left(x_{n}\right)$ is said to be $A$-statistically convergent to $l$ and denoted by st $\operatorname{st}_{A} \lim x=l$ if for every $\varepsilon>0, \delta_{A}\left\{n \in \mathbb{N}:\left|x_{n}-l\right| \geq \varepsilon\right\}=0$ (see $[6,13])$.

Taking $A=C_{1}$, the Cesaro matrix of order one in (3.1), $A$-statistical convergence reduces to statistical convergence $[8,10]$.Taking $A=I$, the identity matrix then $A$ statistical convergence reduces to ordinary convergence. Kolk [9] proved that in the case of $\lim _{j} \max _{n}\left|a_{j, n}\right|=0, A$-statistical convergence is stronger than ordinary convergence.

Now let $A=\left(a_{j n}\right)$ be a non-negative regular summability matrix. Assume that $\left(b_{n}\right)_{n \in \mathbb{N}}$ is a sequence in $[0, \infty)$ satisfying

$$
s t_{A}-\lim _{n} \frac{b_{n}}{n}=0 .
$$

Then we have

$$
s t_{A}-\lim _{n}\left(\frac{b_{n}}{n}\right)^{2}=0
$$

Such a sequence $\left(b_{n}\right)_{n \in \mathbb{N}}$ satisfying (3.2), can be constructed as follows: Take $A=$ $C_{1}$, and define

$$
b_{n}:= \begin{cases}n, & \text { if } n=m^{2}(m \in \mathbb{N}) \\ n^{1 / 3}, & \text { otherwise. }\end{cases}
$$

Then clearly $s t_{C_{1}}-\lim \frac{b_{n}}{n}=s t-\lim \frac{b_{n}}{n}=0$. 
Theorem 2. Let $A=\left(a_{j k}\right)$ be a non-negative regular summability matrix and $\beta>0$ be fixed. If

then

$$
s t_{A}-\lim _{n} \frac{b_{n}}{n}=0
$$

holds for every $f \in E$.

$$
s t_{A}-\lim _{n}\left\|L_{n}^{(\beta)}(f ; x)-f(x)\right\|_{C[0, B]}=0
$$

Proof. Given $r>0$ choose $\varepsilon>0$ such that $\varepsilon<r$. For fixed $\beta>0$, define the following sets:

$$
\begin{aligned}
U & :=\left\{n: \delta_{n}(\beta, B) \geq r\right\}, \\
U_{1} & :=\left\{n: \frac{b_{n}}{n} \geq \frac{r-\varepsilon}{2 N_{f}(\beta, B)}\right\}, \\
U_{2} & :=\left\{n:\left(\frac{b_{n}}{n}\right)^{2} \geq \frac{r-\varepsilon}{2 N_{f}(\beta, B)}\right\},
\end{aligned}
$$

where $N_{f}(\beta, B)$ and $\delta_{n}(\beta, B)$ be the same as in Theorem 1 . Then it is clear that $U \subseteq U_{1} \cup U_{2}$, which gives

$$
\sum_{k \in U} a_{j k} \leq \sum_{k \in U_{1}} a_{j k}+\sum_{k \in U_{2}} a_{j k} .
$$

Letting $j \rightarrow \infty$ in (3.5) and using (3.2) and (3.3), we have $\lim _{j} \sum_{k \in U} a_{j k}=0$. This proves that $s t_{A}-\lim _{n} \delta_{n}(\beta, B)=0$ which also implies

$$
s t_{A}-\lim _{n} \omega_{B+1}\left(f, \delta_{n}^{1 / 2}(\beta, B)\right)=0 .
$$

Using Theorem 1 we get the result.

Remark that choosing the sequence $\left(b_{n}\right)_{n \in \mathbb{N}}$ as in (3.4), the statistical approximation results in Theorem 2 works, however its classical case does not work since $\left(\frac{b_{n}}{n}\right)_{n \in \mathbb{N}}$ is not convergent in the ordinary sense.

\section{REFERENCES}

[1] A. Aral and O. Duman, "A Voronovskaya-type formula for SMK operators via statistical convergence," Math. Slovaca, vol. 61, no. 2, pp. 235-244, 2011.

[2] G. Bretti, P. Natalini, and P. E. Ricci, "Generalizations of the Bernoulli and Appell polynomials," Abstr. Appl. Anal., vol. 2004, no. 7, pp. 613-623, 2004.

[3] O. Doğru and M. Örkcü, "Statistical approximation by a modification of $q$-Meyer-König and Zeller operators," Appl. Math. Lett., vol. 23, no. 3, pp. 261-266, 2010.

[4] O. Duman and C. Orhan, "Rates of $A$-statistical convergence of positive linear operators," Appl. Math. Lett., vol. 18, no. 12, pp. 1339-1344, 2005.

[5] O. Duman and C. Orhan, "Rates of $A$-statistical convergence of operators in the space of locally integrable functions," Appl. Math. Lett., vol. 21, no. 5, pp. 431-435, 2008. 
[6] H. Fast, "Sur la convergence statistique," Colloq. Math., vol. 2, pp. 241-244, 1951.

[7] A. R. Freedman and J. J. Sember, "Densities and summability," Pac. J. Math., vol. 95, pp. 293305, 1981.

[8] J. A. Fridy, "On statistical convergence," Analysis, vol. 5, pp. 301-313, 1985.

[9] E. Kolk, "Matrix summability of statistically convergent sequences," Analysis, vol. 13, no. 1-2, pp. 77-83, 1993.

[10] H. I. Miller, "A measure theoretical subsequence characterization of statistical convergence," Trans. Am. Math. Soc., vol. 347, no. 5, pp. 1811-1819, 1995.

[11] G. M. Mittag-Leffler, "Sur la nouvelle function $E_{\alpha}$, " C. R. Acad. Sci. Paris, vol. 137, p. C.R. Acad. Sci. Paris, 1903.

[12] M. A. Özarslan and H. Aktuğlu, “ $A$-statistical approximation of generalized Szász-Mirakjan-Beta operators,” Appl. Math. Lett., vol. 24, no. 11, pp. 1785-1790, 2011.

[13] H. Steinhaus, "Sur la convergence ordinaire et la convergence asympttique," Colloq. Math., vol. 2, pp. 73-74, 1951.

[14] A. Wiman, "Über den fundamentalsatz in der theorie der funktionen $E_{\alpha}(x)$," Acta Math., vol. 29, pp. 191-201, 1905.

\section{Author's address}

\section{Mehmet Ali Özarslan}

Eastern Mediterranean University, Gazimagusa, TRNC, Mersin 10, Turkey

E-mail address: mehmetali.ozarslan@emu.edu.tr 